\section{Cerebral ventricular size and dyskinesia}

SIR: Following publication of Cooper et als letter (Journal, October 1991, 159, 583-584), we have looked again at the unpublished data in our study. Cortical atrophy had been evaluated 'blind', at the time of the computerised tomography (CT) scans by a consultant neuroradiologist. Scores were given for the frontal, temporal, parietal, and occipital regions, and the scores summed. The total atrophy score was not found to be related to dyskinesia development and was not reported in our paper. We have now reanalysed the frontal scores separately. Frontal atrophy was less correlated with age $(r=0.34)$ than VBRs or total atrophy scores. When age was allowed for, there was a significant correlation between frontal atrophy and dyskinesia development $(r=0.40$; $P=0.03$ ). There was no evidence of an association between neuroleptics and frontal atrophy.

We thank Dr Cooper and his colleagues for their suggestion that we should reanalyse our data. The results given above are preliminary and more appropriate analyses remain to be done. Nevertheless, these results support their hypothesis that frontal cortical atrophy is associated with dyskinesia development and so add to the increasing evidence of frontal lobe deficits in schizophrenia.

\section{H. A. McClelland}

38 Nuns Moor Crescent

Newcastle upon Tyne NE4 9BE

St Nicholas Hospital

T. A. KERR

Gosforth

Newcastle upon Tyne

A. V. Metcalfe

Department of Engineering Mathematics

University of Newcastle upon Tyne

\section{Chronic fatigue syndrome}

SIR: We were interested to read Lynch et al's comments on the problems associated with the term chronic fatigue syndrome (Journal, September 1991, 159,439 ). While we are aware of the shortcomings of the name 'myalgic encephalomyelitis' (ME), we do not regard 'chronic fatigue syndrome' (CFS) as a suitable replacement, for the reasons outlined below.

Firstly, it is difficult to see the advantages of using the same name for such different conditions as ME, tiredness and effort syndrome. It is a little like combining appendicitis, Crohn's Disease and irritable bowel syndrome and referring to all three as abdominal pain syndrome.

Secondly, since the vast majority of individuals suffering from chronic fatigue do not have CFS, the new term is misleading. For instance, a recent study by Manu et al (1991) revealed that only 9 of the 200 patients presenting with chronic fatigue fulfilled the American diagnostic criteria for CFS. Similarly, David et al (1990) reported that out of 70 patients suffering from unexplained chronic fatigue, only one was thought to have CFS. Yet despite the obvious differences in severity and aetiology, a number of writers have generalised research findings from the former to the latter (e.g. Stewart, 1990).

The confusion between the two has also encouraged the trivialisation of ME, with 'tiredness' replacing references to exhaustion (Welsby, 1990) and writers claiming that it is not a disease but merely 'a human predicament' (Hodgkinson, 1991). As for the comment that patients are people who 'expect to feel $100 \%, 100 \%$ of the time' (Welsby, 1990), this is not only rather unkind but also unjustified in most cases of ME.

Unfortunately, the new UK consensus criteria will make matters worse. Apart from their 'poor predictive validity', they are broader than those used in other countries where CFS generally corresponds to what is known as ME. Indeed, there are no references to any symptoms which would differentiate CFS from chronic fatigue. Consequently, patients diagnosed with CFS in Britain form a much more heterogeneous population than CFS patients from America and Australia and it is therefore difficult to compare research.

The failure to distinguish ME from unexplained chronic fatigue has also complicated the evaluation of treatment. For example, while graded exercise and cognitive therapy appear to be helpful in patients diagnosed according to British criteria (Butler et al, 1991), this regime was no better than another ineffective treatment when recently tested in a sample of patients selected according to the much more stringent Australian definition (Hickie, unpublished).

We hope that this letter provides a basis for further discussion and that it will not be too long before ME is once more accepted as a clinical entity in its own right.

Butler, S., Chalder, T., Ron, M., et al (1991) Cognitive behaviour therapy in chronic fatigue syndrome. Journal of Neurology. Neurosurgery and Psychiatry, 54, 153-158.

DA VID, A., Pelosi, A., McDonald, E., et al (1990) Tired, weak, or in need of rest: fatigue among general practice attenders. British Medical Journal, 310, 1199-1202.

Hodgkinson, N. (1991) ME epidemic of anger. Sunday Express, February 3, 14

Manu, P., Matthews, D. A. \& Lane, T. J. (1991) Panic disorder among patients with chronic fatigue. Southern Medical Journal, $84,451-456$

STEWART, D. E. (1990) Emotional disorders misdiagnosed as physical illness: environmental hypersensitivity, candidiasis 